\title{
Fermented papaya preparation halts the progression of non-alcoholic steatohepatitis in rats
}

\author{
Shinki Murakami ${ }^{1,2,3}$, Fusako Takayama ${ }^{2}$, Toru Egashira ${ }^{2}$, Mitsuko Imao $^{3}$, Akitane Mori $^{1}$ \\ ${ }^{1}$ Department of Brain Science, Graduate School of Medicine, Dentistry and Pharmaceutical Sciences, Okayama University, Okayama, \\ Japan \\ ${ }^{2}$ Department of Clinical Pharmaceutical Science, Graduate School of Medicine, Dentistry and Pharmaceutical Sciences, Okayama \\ University, Okayama, Japan \\ ${ }^{3}$ SAIDO Corporation, Fukuoka, Japan; ${ }^{*}$ Corresponding Author: takayamf@cc.okayama-u.ac.jp
}

Received 27 March 2013; revised 29 April 2013; accepted 9 May 2013

Copyright (C) 2013 Shinki Murakami et al. This is an open access article distributed under the Creative Commons Attribution License, which permits unrestricted use, distribution, and reproduction in any medium, provided the original work is properly cited.

\begin{abstract}
Non-alcoholic steatohepatitis (NASH) can progress to cirrhosis or hepatocellular carcinoma. Oxidative stress is implicated in NASH progression. Fermented papaya preparation (FPP) has oxygen radical scavenging activity and is effective in oxidative stress-related diseases. We investigated the effects of FPP on NASH progression using a rat NASH model. Plasma biochemical parameters and lipid peroxidation in the liver were elevated in NASH rats. Histologically, the liver of NASH rats showed liver fibrosis, mitochondrial dysfunction and over-expression of microsomal CYP2E1. Myeloperoxidase activity and nuclear factor-kappaB activation were also markedly increased in NASH. Oral administration of FPP ameliorated these changes in NASH rats. These results suggest that FPP halts NASH progression through its anti-oxidative and antiinflammatory properties.
\end{abstract}

Keywords: Fermented Papaya Preparation; Non-Alcoholic Steatohepatitis; Oxidative Stress; Antioxidant Activity; Inflammation

\section{INTRODUCTION}

Non-alcoholic fatty liver disease (NAFLD) is the most frequent cause of liver damage, closely associated with obesity and the metabolic syndrome, and often progresses to non-alcoholic steatohepatitis (NASH). NASH has attracted attention in recent years based on its relation with cirrhosis and hepatocellular carcinoma. The pathological findings in NASH are similar to those found in alcoholic steatohepatitis; including liver inflammation, necrosis, apoptosis, and fibrosis, despite the lack of alcohol consumption [1]. There is no effective drug therapy for prevention or treatment of NASH. Therefore, development of novel supplements or drugs with preventive effect for NASH is desirable. The pathogenesis of NASH is not clear, but the "two hit theory" is the most accepted [2]. It is based on fat accumulation in hepatocytes as a first hit, followed by the second hit of oxidative stress (OS), inflammatory cytokines, and insulin resistance.

We previously established a clinically relevant rat model of NASH (Patent application No. PCT/JP2007/52477) [3]. This model is prepared as follows; rats are fed a choline-deficient high-fat diet for 4 weeks to induce steatosis, and are treated by intraperitoneal injections of nitrite for 6 weeks to induce hypoxia-related oxidative stress. Using this model, it has been demonstrated that OS causes extensive hepatic fibrosis, and that these effects can be ameliorated by anti-oxidants, such as fermented green tea extract [4], vitis coignetiae pulliat leaves [5,6], docosahexaenoic acid [7], and spirulina [8].

Fermented papaya preparation (FPP) is produced by fermentation of unripe papaya (Carica papaya Linn.) using a variety of enzymes. Nutritional analysis has demonstrated the presence of beneficial amino acids, some vitamins, and various trace metals, such as iron, calcium, magnesium, copper and zinc in FPP. Papaya is widely known as a traditional medicinal fruit with beneficial properties, such as the antioxidant activity $[9,10]$, heaptoprotective activity against $\mathrm{CCl}_{4}[11]$ and enhancement of phase II enzyme activity, such as glutathione S-transferase in hepatocytes [12]. Previous studies reported that FPP has radical scavenging activity $[13,14]$ as well as protection against diseases related to oxidative stress, such as traumatic epilepsy [14-16], Alzheimer's disease [17], contact hypersensitivity [18], and stress-induced gastric 
mucosal lesions [19].

The present study was designed to determine the effects of FPP on prevention of NASH progression, and to elucidate the mechanism of the action.

\section{MATERIALS AND METHODS}

\subsection{Chemicals}

FPP was obtained from SAIDO Corporation (Fukuoka, Japan). 5,5-Dimethyl-1-pyrroline N-oxide (DMPO) was purchased from Labotech Co. (Tokyo, Japan). Dodecyl maltoside (DM) was purchased from Dojin Laboratories (Kumamoto, Japan). Benzamidine hydrochloride and sodium nitrate were purchased from Nacalai Tesque Inc. (Kyoto, Japan). $\beta$-NADH was purchased from Oriental Yeast Co. (Osaka, Japan). Other reagents were obtained from Wako Pure Chemical Industries (Osaka). All chemicals used in this study were of the highest grade available.

\subsection{Animals}

Six-week-old male Wistar rats weighting $160-170 \mathrm{~g}$ were purchased from Shimizu Experimental Animals (Shizuoka, Japan). The rats were housed in filter-protected cages set at $23^{\circ} \mathrm{C}$ and ambient light was controlled automatically to produce a light/dark $12 \mathrm{~h}$ cycle. The Animal Ethics Committee of Okayama University approved the study. All animal procedures described in this study were in strict accordance with the Guidelines for Animal Experiments at Okayama University Medical School.

\subsection{Experimental Design}

We induced fatty liver in rats by feeding them a choline-deficient high-fat (CDHF) diet (Oriental Yeast, Tokyo) for 4 weeks. The control group $(n=6)$ was fed the standard chow (Oriental Yeast Co.). The CDHF group (n =6) was fed CDHF diet only through the experiment period of 10 weeks. NASH group $(n=6)$ was fed CDHF diet, followed by injection of sodium nitrite $30 \mathrm{mg} / \mathrm{kg}$ for 6 weeks from the 5th week [3]. In the NASH + PL (low dose of FPP: $1 \%$ solution) group $(n=6)$ and NASH + PH (high dose of FPP: 3\% solution) group $(n=6)$ were orally administered FPP solution in drink in water for 6 weeks from 5th week. After the experiment period, the rats were fasted overnight and sacrificed by anesthetizing with diethyl ether. Tissue samples were prepared to determine oxidative injury and effects of FPP, by using biochemical and OS markers and assessment of histological changes. The liver samples were fixed in $20 \%$ formalin or snap-frozen in liquid nitrogen for histological examination and for analyses of lipid peroxidation and antioxidant activity.

\subsection{Histological Examination}

Paraffin-embedded tissue sections were cut (4- $\mu \mathrm{m}$ thick) and stained with Masson trichrome for fibrosis. Tissue morphology was assessed under an Olympus light microscope (Olympus, Tokyo).

\subsection{Biochemical Analyses}

The activities of alanine aminotransferase (ALT) and aspartate aminotransferase (AST) in plasma were determined by commercial enzyme assay kits (Wako Pure Chemical Industries, Osaka). The level of plasma alkaline phosphatase (ALP) was determined using a standard assay.

\subsection{Reactive Oxygen Species (ROS) in Rat Liver Mitochondria}

Liver mitochondria were prepared by differential centrifugation of the tissue homogenates. In brief, rats were transcardially perfused with ice-cooled $1.15 \% \mathrm{KCl}$ buffer containing $5 \mathrm{mM}$ benzamidine ( $\mathrm{pH}$ 7.4) via the inferior vena cava [4]. The liver tissue was then removed and homogenized in $5 \mathrm{mM}$ Tris- $\mathrm{HCl}$ buffer (pH 7.4) containing $0.25 \mathrm{mM}$ sucrose and $100 \mathrm{mM} \mathrm{KCl}$. The mixture was centrifuged at $2500 \mathrm{rpm}$ for $10 \mathrm{~min}$. The supernatant was collected and centrifuged at $9000 \times \mathrm{g}$ for $20 \mathrm{~min}$. The resulting pellet containing the mitochondrial fraction was resuspended in the buffer and stored at $-80^{\circ} \mathrm{C}$ until analysis. The protein concentration was determined using a BCA kit (Pierce, Rockford, IL) with bovine serum albumin as a standard. Electron spin resonance (ESR) technique with DMPO was used to determine ROS generation in liver mitochondria. Rat liver mitochondria isolates $(500 \mu \mathrm{g})$ were mixed in $0.1 \mathrm{ml}$ potassium phosphate buffer ( $\mathrm{pH} 7.4$ ) containing $0.1 \% \mathrm{DM}, 5 \mathrm{mM}$ glutamate, 5 $\mathrm{mM}$ malate, $0.1 \mathrm{mM}$ succinate, $0.92 \mathrm{mM}$ DMPO, and 0.1 $\mathrm{mM}$ NADH, incubated for $5 \mathrm{~min}$ at $37^{\circ} \mathrm{C}$ and then measured. Analyses were performed at room temperature under ambient atmosphere. Using a manganese signal as an internal standard, the relative peak height of the second signal obtained from the DMPO-OH adducts was evaluated. The conditions for ESR measurements were as follows; microwave power: $8 \mathrm{~mW}$, modulation amplitude width: $0.1 \mathrm{mT}$, response time: $0.1 \mathrm{~s}$, scanning time: $4 \mathrm{~min}$, magnetic field: $336.0 \pm 5 \mathrm{mT}$ (JES-RE1X/HR, JEOL, Tokyo).

\subsection{Western Blot Analysis}

The levels of microsomal cytochrome P450 2E1 (CYP2E1) and nuclear factor kappa B (NF- $\mathrm{kB})$ in the liver were analyzed by western blotting. The samples were solubilized in $0.5 \mathrm{M}$ Tris-HCL $(\mathrm{pH} 6.8)$ with sodium dodecyl sulfate (SDS), 10\% glycerol, 5\% 2-mer- 
captoethanol and $0.05 \%$ bromophenol blue, then degenerated by incubation at $100^{\circ} \mathrm{C}$ for $5 \mathrm{~min}$. The samples were subjected to SDS-10\% polyacrylamide gel electrophoresis. After electrophoresis, proteins are transferred to polyvinylidene fluoride membrane. Then, the non-specific binding proteins were blocked in 5\% nonfat milk dissolved in TBS-T (Tween-20 in Tris-buffered saline, $\mathrm{pH}$ 7.4) for 1 hour and incubated with primary rabbit anti-human/rat CYP2E1 polyclonal antibodies (1:1500; Chemicon International, Temecula, CA) or mouse antihuman/rat NF- $\kappa \mathrm{B}$ monoclonal for 1 hour, and the membranes were incubated with secondary anti-rabbit or mouse IgG antibody (1:5000; Santa Cruz Biotechnology, Santa Cruz, CA) for 1 hour. $\beta$-actin or histon was used to assess equal protein loading. Protein bands were visualized using enhanced Chemiluminescence Luminol Reagent (Santa Cruz Biotechnology).

\subsection{Lipid Peroxidation Assay}

The liver homogenate supernatants collected from centrifugation of $30 \mathrm{mg} / \mathrm{ml}$ liver homogenate in $120 \mathrm{mM}$ $\mathrm{KCl}-30 \mathrm{mM}$ phosphate buffer ( $\mathrm{pH} 7.4$ ), were mixed with Luminol $(130 \mu \mathrm{g} / \mathrm{ml})$ and analyzed in an Auto Lumat device (Tristar LB941, Berthold Technologies, Germany), at $37^{\circ} \mathrm{C}$ for $5 \mathrm{~min}$. Then, $0.33 \mathrm{mM}$ tert-butyl hydroperoxide $(t-\mathrm{BuOOH})$ was added, and the chemiluminescence (CL) intensity was detected for $120 \mathrm{~min}$ along with incubation at $37^{\circ} \mathrm{C}$. Data were expressed as mean \pm SEM of the cumulative CL intensity for $120 \mathrm{~min}$.

\subsection{Myeloperoxidase Activity}

Myeloperoxidase (MPO) activity was used as an index of neutrophil infiltration in the liver. The liver was homogenated in $0.3 \mathrm{M}$ sucrose containing $0.22 \%$ cetyltrimethyl ammonium and $10 \mathrm{mM}$ citirate $(\mathrm{pH} 5.0)$ and sonicated. The homogenates were centrifuged at $6500 \times$ $\mathrm{g}$ for $20 \mathrm{~min}$. The supernatants were reacted with substrate solution ( $3 \mathrm{mM}$ tetramethylbenzidine, $120 \mu \mathrm{M}$ resorcinol, and $2.2 \mathrm{mM} \mathrm{H}_{2} \mathrm{O}_{2}$ ) in shading and mixed with 4 $\mathrm{N} \mathrm{H}_{2} \mathrm{SO}_{4}$, followed by reading absorbance at $450 \mathrm{~nm}$.

\subsection{Statistical Analysis}

Values were expressed as mean \pm standard error of the mean (SEM) of 4 - 6 rats. Differences among groups were examined by one-way analysis of variance (ANOVA) with Tukey test. A $P$ value less than 0.05 was considered statistically significant.

\section{RESULTS}

\subsection{Effects of FPP on Liver Histology}

Figure 1 shows microphotographs of histological changes in a representative animal. Macrovesicular hepatic steatoses were evident in the liver of CDHF-treated rats, and marked fibril formations were observed in the liver of NASH rats by Masson-trichrome staining. However, these histological changes were attenuated in FPP-treated rats, and the effect was concentration-dependent.

\subsection{Effects of FPP on Biochemical Parameters}

Plasma ALT, AST and ALP activities were significantly higher in NASH rats than the control. Plasma ALT activity was lower in both PL and PH groups, though the difference was not significant $(P>0.05$, Figure 2). Plasma AST activity was significantly improved in the PH group compared with NASH $(P<0.05)$. Moreover, plasma ALP activity was significantly better in both PL and $\mathrm{PH}$ groups compared with NASH $(P<0.01)$.

\subsection{Effects of FPP on Mitochondrial ROS Generation in the Liver}

ROS generation from liver mitochondria was estimated by ESR analysis (Figure 3). ROS generation was significantly higher in NASH compared with the control $(P<0.05)$. ROS generation was comparable in the PL and NASH groups, but significantly reduced in the $\mathrm{PH}$ group $(P<0.05)$.

\subsection{Effects of FPP on Microsomal CYP2E1 Induction in the Liver}

To investigate the source of ROS in NASH, we measured liver microsomal CYP2E1 expression by western blotting. CYP2E1 was significantly induced in the NASH, compared with the control $(P<0.01$, Figure 4). However, CYP2E1 induction was significantly inhibited in the PH group $(P<0.05)$.

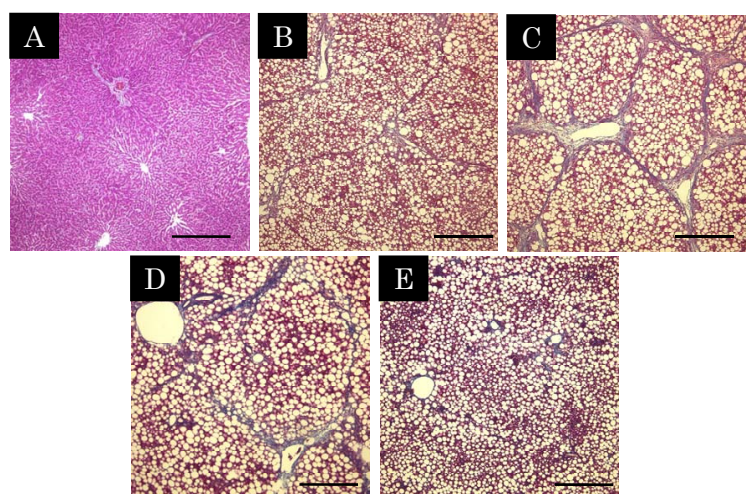

Figure 1. Histopathological changes in the liver by Massontrichrome stain (A - E). The blue parts represent the collagenous region. A; Control, B; CDHF, C; NASH, D; NASH + $\mathrm{PL}, \mathrm{E} ; \mathrm{NASH}+\mathrm{PH}$. Scale Bar $=500 \mu \mathrm{m}$. 

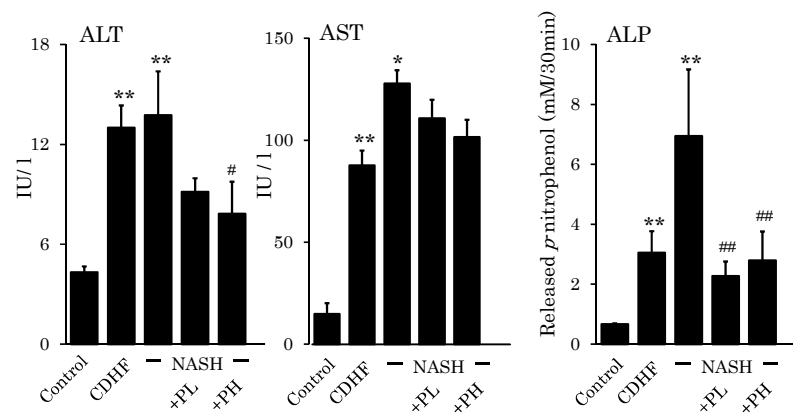

Figure 2. Effects of FPP on biochemical parameters. Data are mean \pm SEM of $4-6$ rats. ${ }^{*} p<0.05,{ }^{* *} p<0.01$, compared with the Control, ${ }^{\#} p<0.05,{ }^{\#} p<0.01$, compared with NASH (ANOVA with Tukey-test).

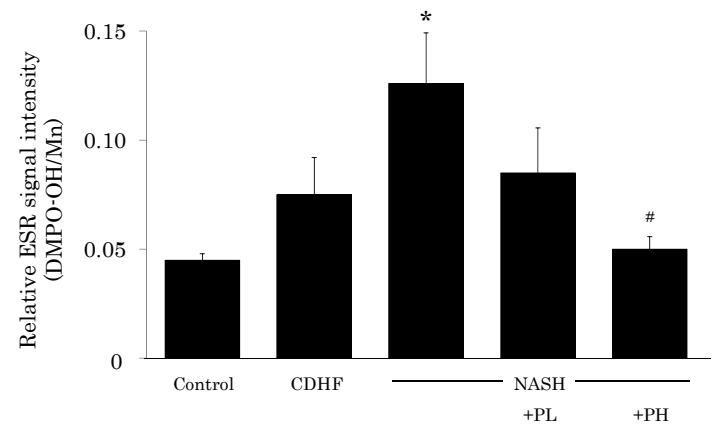

Figure 3. Effects of FPP on liver mitochondrial ROS generation. Data are mean \pm SEM of $4-6$ rats. ${ }^{*} p<0.05, p<0.01$, compared with Control, ${ }^{\#} p<0.05,{ }^{\#} p<0.05$, compared with NASH (ANOVA with Tukey-test).

\subsection{Effects of FPP on Lipid Peroxidation in the Liver}

We measured lipid peroxidation as an index of OS status in the liver, using CL method. Lipid peroxidation was significantly higher in NASH rats compared to the control $(P<0.01$, Figure 5). However, administration of $3 \%$ FPP solution resulted in reduction of lipid peroxidation $(P<0.05)$.

\subsection{Effects of FPP on MPO Activity in the Liver}

MPO activity in the liver was significantly increased in the liver of NASH (i.e., higher neutrophil infiltration), compared with the control $(P<0.01$, Figure 6). However, administration of $3 \%$ FPP solution resulted in reduction of MPO activity $(P<0.05)$.

\subsection{Effects of FPP on NF-kB Activation}

The level of activated NF- $\mathrm{kB}$ in the nuclei of hepatocytes was markedly increased in NASH rats compared with the control $(P<0.01)$. However, the level was significantly decreased in $\mathrm{PH}$ rats $(P<0.05$, Figure 7$)$.

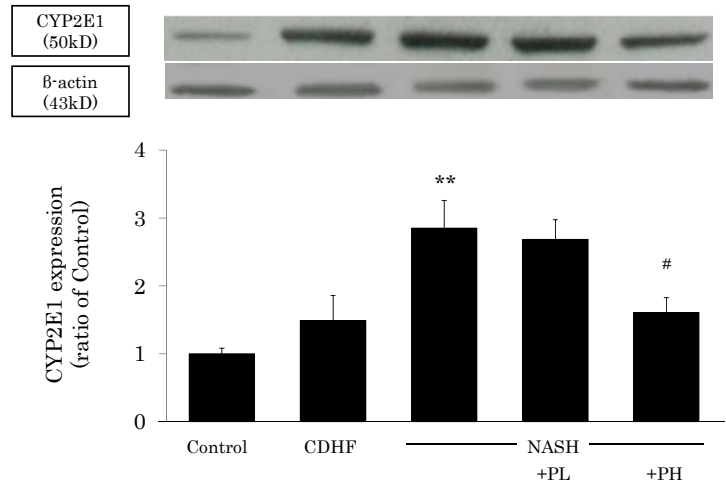

Figure 4. Effects of FPP on liver microsomal CYP2E1 induction. Data are mean \pm SEM of $4-6$ rats. ${ }^{* *} p<0.01$, compared with Control, ${ }^{*} p<0.05$, compared with NASH (ANOVA with Tukey-test).

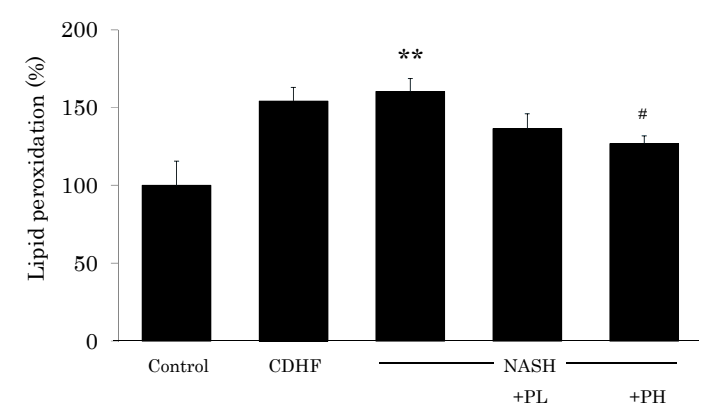

Figure 5. Effects of FPP on lipid peroxidation in the liver. Data are mean \pm SEM of $4-6$ rats. ${ }^{* *} p<0.01$, compared with the Control, ${ }^{\#} p<0.05$, compared with NASH (ANOVA with Tukey-test).

\section{DISCUSSION}

The present study demonstrated the preventive effects of oral FPP administration on NASH progression. Similar to previous reports, high plasma ALT, AST and ALP activities, together with steatosis and fibril formation in the liver were confirmed in our rat NASH model, similar to human NASH pathology. In this study, oral administration of FPP markedly reduced these changes.

OS is regarded as the "second hit" in the development of NASH. Thus, it is postulated that anti-oxidants can prevent NASH progression. Indeed, previous studies reported that patients with NASH suffered depletion of antioxidants such as glutathione, vitamins $\mathrm{C}$ and $\mathrm{E}$ [2022]. Mitochondrial $\beta$-oxidation can be a major source of ROS, which contribute to the progression of NASH [2325]. Such process is promoted by accumulation of fat in the liver, resulting in mitochondrial dysfunction. Consequently, mitochondrial dysfunction seems to be important in the pathogenesis of NASH. In the present study, ROS generation from the liver mitochondria was increased in NASH rats. No change was found in the PL group, whereas the $\mathrm{PH}$ group showed attenuation of 


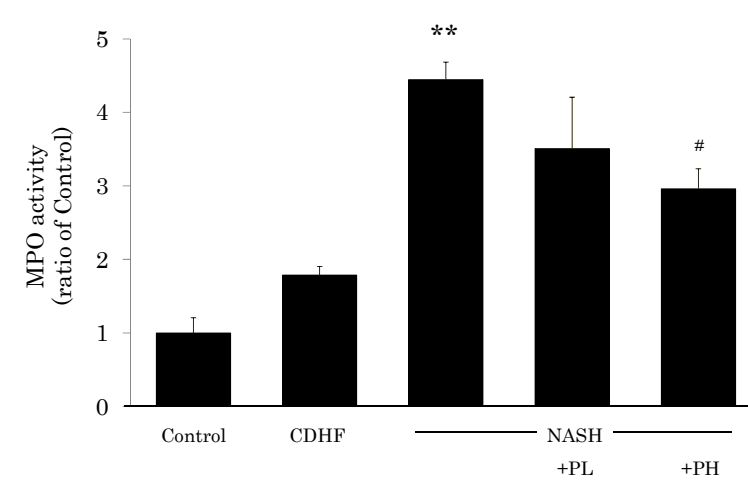

Figure 6. Effects of FPP on MPO activity in the liver. Data are mean \pm SEM of $4-6$ rats. ${ }^{* *} p<0.01$, compared with the Control, ${ }^{\#} p<0.05$, compared with NASH (ANOVA with Tukey-test).

increased ROS generation (64.1\%). These results suggest that FPP can improve mitochondrial dysfunction and potentially halt NASH progression.

Excess of free fatty acids can cause the induction of microsomal CYP2E1, which results in ROS overproducetion and lipid peroxidation in the liver [26]. Thus, mi crosomal CYP2E1 overexpression is also considered to play a role as the source of ROS in NASH liver. In fact, previous studies suggested that oxidative stress may play a major role in the activation of hepatic stellate cells in NASH [27,28]. Lipid peroxidation has also been shown to stimulate collagen production in fibroblasts and hepatic stellate cells [29]. Hepatic lipid peroxidation may lead to the formation of cytotoxic intermediates, which may cause cellular injury directly, or indirectly through the initiation of a series of inflammatory responses. The present study demonstrated significant increase in liver microsomal CYP2E1 expression and lipid peroxidation in NASH, compared to the control. Administration of FPP significantly suppressed CYP2E1 induction and lipid peroxidation. Based on these findings, it seems that FPP can ameliorate NASH progression by suppression of excess ROS production from microsomal CYP2E1 followed by lipid peroxidation in the liver.

MPO activity has been used as an index of neutrophil infiltration in inflammatory tissues. These cells produce ROS, further worsening the progression of human NASH. In the present study, NASH rats showed significant increase in liver MPO activity, which could contribute to oxidative stress as second hit. In this regard, NF- $\mathrm{\kappa B}$ is highly activated at sites of inflammation by ROS or inflammatory stimuli and can induce transcription of proinflammatory cytokines, chemokines, adhesion molecules, matrix metalloproteinase, COX-2, and inducible nitric oxide synthase (iNOS) [30]. Furthermore, antioxi dants have been shown to inhibit NF- $\mathrm{KB}$ activation and hence, NF-B has been characterised as a redox sensor [30]. NF- $\mathrm{KB}$ is also described as an important proinfflammatory mediator of lesion development in dietary

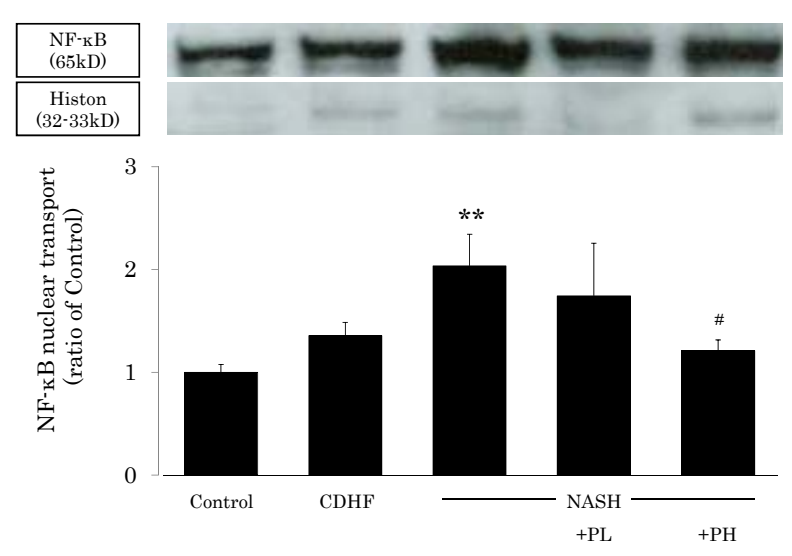

Figure 7. Effects of FPP on NF- $\kappa$ B activity in the liver. Data are mean \pm SEM of $4-6$ rats. ${ }^{* *} p<0.01$, compared with the Control, ${ }^{\#} p<0.05$, compared with NASH (ANOVA with Tukey-test).

murine model of steatohepatitis [31]. Also, recent work demonstrates that free fatty acids can induce the activation of key regulator of inflammation, NF- $\mathrm{kB}$. Activation of NF- $\mathrm{\kappa B}$ in NASH liver is accompanied by increased induction of iNOS [32], which is a transcriptional target for NF-kB. Nitric oxide (NO) forms peroxynitrite in the presence of superoxide radicals in certain pathological conditions, such as inflammation and ischemia reperfusion injury. Another study hypothesized that iNOS-derived NO increases and acts as a second hitter in NASH rats fed choline-deficient, l-amino acid-defined diet [33]. Thus, NO derived through iNOS is involved in the induction of inflammation, apoptosis, and fibrosis in the liver, and possibly contribute to NASH progression. Our results suggest that FPP reduced liver inflammation that was induced NF- $\mathrm{\kappa B}$ activation by suppressing oxidative stress, such as mitochondrial dysfunction and overexpression of microsomal CYP2E1.

In conclusion, the present study demonstrated that oral administration of FPP halted NASH progression, and that the effects of FPP involve anti-oxidative and anti-inflammatory properties.

\section{REFERENCES}

[1] Ludwig J., Viggiano, T.R., McGill, D.B. and Oh, B.J. (1980) Mayo Clinic experiences with a hitherto unnamed disease. Mayo Clinic Proceedings, 55, 434-438.

[2] Day, C.P. and James, O. (1998) Steatohepatitis: A tale of "two hits"? Gastroenterology, 114, 842-845. doi:10.1016/S0016-5085(98)70599-2

[3] Takayama, F., Hobara, N., Nakamoto, K., Ohro, M., Yoshida, N., Takeda, A., Kawasaki, H. and Egashira, T. (2006) Construction of a non-alcoholic steatohepatitis model induced by fatty liver and nitrite administration. Journal of Pharmacological Science, 100, 164.

[4] Kazuo, N., Fusako, T., Mitsumasa, M., Yuki, H., Toru, E., 
Tetsuya, O., Hiromu, K. and Akitane, M. (2009) Beneficial effects of fermented green tea extract in a rat model of non-alcoholic steatohepatitis. Journal of Clinical Biochemistry \& Nutrition, 44, 239-246. doi:10.3164/jcbn.08-256

[5] Fusako, T., Kazuo, N., Hiromu, K., Mitsumasa, M., Toru, E., Keiji, U., Azusa, H., Shigeru, O. and Akitane, M. (2009) Beneficial effects of vitis coignetiae pulliat leaves on nonalcoholic steatohepatitis in a rat model. Acta Medicne Okayama, 63, 105-111.

[6] Pak, W., Takayama, F., Hasegawa, A., Mankura, M., Egashira, T., Ueki, K., Nakamoto, K., Kawasaki, H. and Mori, A. (2012) Water extract of Vitis coignetiae Pulliat leaves attenuates oxidative stress and inflammation in progressive NASH rats. Acta Medicine Okayama, 66, 317-327.

[7] Takayama, F., Nakamoto, K., Totani, N., Yamanushi, T., Kabuto, H., Kaneyuki, T. and Mankura, M. (2010) Effects of docosahexaenoic acid in an experimental rat model of nonalcoholic steatohepatitis. Journal of Oleo Science, 59, 407-414. doi:10.5650/jos.59.407

[8] Pak, W., Takayama, F., Mine, M., Nakamoto, K., Kodo, Y., Mankura, M., Egashira, T., Kawasaki, H. and Mori, A. (2012) Anti-oxidative and anti-inflammatory effects of spirulina on rat model of non-alcoholic steatohepatitis. Journal of Clinical Biochemistry \& Nutrition, 51, 227-234.

[9] Osato, J.A., Santiago, L.A., Remo, G.M., Cuadra, M.S. and Mori, A. (1993) Antimicrobial and antioxidant activities of unripe papaya. Life Science, 53, 1383-1389. doi:10.1016/0024-3205(93)90599-X

[10] Mehdipour, S., Yasa, N., Dehghan, G., Khorasani, R., Mohammadirad, A., Rahimi, R. and Abdollahi, M. (2006) Antioxidant potentials of Iranian Carica papaya juice in vitro and in vivo are comparable to alpha-tocopherol. Phytother Research, 20, 591-594. doi:10.1002/ptr.1932

[11] Rajkapoor, B., Jayakar, B., Kavimani, S. and Murugesh, N. (2002) Effect of dried fruits of Carica papaya Linn on hepatotoxicity. Biological \& Pharmaceutical Bulletin, 25, 1645-1646. doi:10.1248/bpb.25.1645

[12] Nakamura, Y., Morimitsu, Y., Uzu, T., Ohigashi, H., Murakami, A., Naito, Y., Nakagawa, Y., Osawa, T. and Uchida, K. (2000) A glutathione S-transferase inducer from papaya: Rapid screening, identification and structure-activity relationship of isothiocyanates. Cancer Letters, 157, 193-200. doi:10.1016/S0304-3835(00)00487-0

[13] Noda, Y., Murakami, S., Mankura, M. and Mori, A. (2008) Inhibitory effect of fermented papaya preparation on hydroxyl radical generation from methylguanidine. Journal of Clinical Biochemistry \& Nutrition, 43, 185-190. doi:10.3164/jcbn.2008062

[14] Imao, K., Wang, H., Komatsu, M. and Hiramatsu, M. (1998) Free radical scavenging activity of fermented papaya preparation and its effect on lipid peroxide level and superoxide dismutase activity in iron-induced epileptic foci of rats. Biochemistry \& Molecular Biology International, 45, 11-23.

[15] Imao, K., Komatsu, M., Wang, H. and Hiramatsu, M. (1999) Inhibitory effect of fermented papaya preparation on oxidative DNA damage and tissue injury in the brain formed during iron-induced epileptogenesis in rats. Journal of
Brain Science, 25, 71-77.

[16] Mori, A., Yokoi, I., Noda, Y. and Willmore, L.J. (2004) Natural antioxidants may prevent posttraumatic epilepsy: A proposal based on experimental animal studies. Acta Medicine Okayama, 58, 111-118.

[17] Zhang, J., Mori, A., Chen, Q. and Zhao, B.L. (2006) Fermented papaya preparation attenuates $\beta$-amyloid precursor protein: $\beta$-amyloid-mediated copper neurotoxicity in $\beta$-amyloid precursor protein and in $\beta$-amyloid precursor Swedish mutation overexpressing SH-SY5Y cells. Neuroscience, 143, 63-72.

doi:10.1016/j.neuroscience.2006.07.023

[18] Hiramoto, K., Imao, M., Sato, E., Inoue, M. and Mori A. (2008) Effect of fermented papaya preparation on dermal and intestinal mucosal immunity and allergic inflammations. Journal Science of Food Agriculture, 88, 11511157. doi:10.1002/jsfa.3190

[19] Shinki, M., Fusako, T., Toru, E., Mitsuko, I. and Akitane, M. (2012) Protective effect of fermented papaya preparation on stress-induced acute gastric mucosal lesion. Journal of Biophysical Chemistry, 3, 311-316. doi:10.4236/jbpc.2012.34038

[20] Leclercq, I.A. (2004) Antioxidant defence mechanisms: New players in the pathogenesis of non-alcoholic steatohepatitis? Clinical Science, 106, 235-237. doi: $10.1042 / \mathrm{CS} 20030368$

[21] Das, K.S., Balakrishnan, V., Mukherjee, S. and Vasudevan, D.M. (2008) Evaluation of blood oxidative stress-related parameters in alcoholic liver disease and non-alcoholic fatty liver disease. Scandinavian Journal of Clinical \& Laboratory Investigation, 68, 323-334. doi: $10.1080 / 00365510701673383$

[22] Harrison, S.A., Torgerson, S., Hayashi, P., Ward, J. and Schenker, S. (2003) Vitamin E and vitamin C treatment improves fibrosis in patients with nonalcoholic steatohepatitis. American Journal of Gastroenterology, 98, 24852490. doi:10.1111/j.1572-0241.2003.08699.x

[23] Sanyal, A.J., Campbell-Sargent, C., Mirshahi, F., Rizzo, W.B., Contos, M.J., Sterling, R.K., Luketic, V.A., Shiffman, M.L. and Clore, J.N. (2001) Nonalcoholic steatohepatitis: Association of insulin resistance and mitochondrial abnormalities. Gastroenterology, 120, 1183-1192. doi:10.1053/gast.2001.23256

[24] Serviddio, G., Sastre, J., Bellanti, F., Viña, J., Vendemiale, G. and Altomare, E. (2007) Mitochondrial involvement in non-alcoholic steatohepatitis. Molecular Aspects Medicine, 29, 22-35. doi:10.1016/j.mam.2007.09.014

[25] Kojima, H., Sakurai, S., Uemura, M., Fukui, H., Morimoto, H. and Tamagawa, Y. (2007) Mitochondrial abnormality and oxidative stress in nonalcoholic steatohepatitis. Alcoholism: Clinical and Experimental Research, 31, 6166. doi:10.1111/j.1530-0277.2006.00288.x

[26] Robertson, G., Leclercq, I. and Farrell, G.C. (2001) Nonalcoholic steatosis and steatohepatitis. II. Cytochrome P450 enzymes and oxidative stress. American Journal of Physiology Gastrointest Liver Physiology, 281, 1135-1139.

[27] Nieto, N., Friedman, S.L. and Cederbaum, A.I. (2002) Cytochrome P450 2E1-derived reactive oxygen species me- 
diate paracrine stimulation of collagen I protein synthesis by hepatic stellate cells. Journal of Biological Chemistry, 22, 9853-9864. doi:10.1074/jbc.M110506200

[28] Nieto, N., Friedman, S.L. and Cederbaum, A.I. (2001) Stimulation and proliferation of primary rat hepatic stellate cells by cytochrome P450 2E1-derived reactive oxygen species. Hepatology, 35, 62-73. doi:10.1053/jhep.2002.30362

[29] George, J., Pera, N., Phung, N., Leclercq, I., Yun Hou, J. and Farrell, G. (2003) Lipid peroxidation, stellate cell activation and hepatic fibrogenesis in a rat model of chronic steatohepatitis. Journal of Hepatology, 39, 756-764.

[30] Tak, P.P. and Firestein, G.S. (2001) NF-kappaB: A key role in inflammatory diseases. Journal of Clinical Investment, 107, 7-11. doi:10.1172/JCI11830

[31] Dela Peña, A., Leclercq, I., Field, J., George, J., Jones, B. and Farrell, G. (2005) NF-kappaB activation, rather than TNF, mediates hepatic inflammation in a murine dietary model of steatohepatitis. Gastroenterology, 129, 16631674. doi:10.1053/j.gastro.2005.09.004

[32] Gao, Y., Song, L.X., Jiang, M.N., Ge, G.Y. and Jia, Y.J. (2008) Effects of traditional Chinese medicine on endotoxin and its receptors in rats with non-alcoholic steatohepatitis. Inflammation, 31, 121-132. doi:10.1007/s10753-008-9057-3

[33] Fujita, K., Nozaki, Y., Yoneda, M., Wada, K., Takahashi, H., Kirikoshi, H., Inamori, M., Saito, S., Iwasaki, T., Terauchi, Y., Maeyama, S. and Nakajima, A. (2010) Nitric oxide plays a crucial role in the development/progression of nonalcoholic steatohepatitis in the choline-deficient, 1-amino acid-defined diet-fed rat model. Alcoholism: Clinical and Experimental Research, 34, 18-24. doi:10.1111/j.1530-0277.2008.00756.x 